\title{
Yields and more
}

\section{Agricultural production is a key point of social vulnerability to climate change, and also a major contributor to those very changes.}

The global trend towards urbanization means that many of us are emotionally distant from farming. Our daily experiences are insulated from the work, the sheer scale and the technical and management effort required to grow food, fibre and, increasingly, fuel for a global population of 7 billion people and counting. Of course, emotional distance has little bearing on our underlying material dependence on farming systems. But it does perhaps limit our perception of the many ways in which agricultural practices influence, and are in turn influenced by, the environment and, by extension, our daily lives. In a Web focus this month we present a collection of research papers and News \& Views pieces, including new research published in this issue and a selection from our archive. These address important themes in our understanding of climate change impacts on agriculture, the influence of agriculture on the climate and our capacity to adapt to what is certain - change.

First and foremost, farming provides the food we eat. Whether people have access to enough food is determined by far more than whether enough calories can be grown, but that is undoubtedly an important starting point. With global populations rising sharply (a medium estimate is 9 billion by 2050; http://esa.un.org/wpp) and climate change threatening to reduce yields, or at least undermine yield gains, even meeting the basic calorie demand of the future is not certain. For this reason there has been a great deal of work to estimate the potential impact of climate change on agricultural yields. In a Commentary (Nature Clim. Change 1, 175-177; 2011) Rotter et al. argued that process-based crop models - the major tool used to estimate climate change impacts on yields - were not fully fit for purpose. Efforts to improve these models and evaluate their relative performance, such as the Agricultural Model Intercomparison and Improvement Project (www.agmip.org), are now beginning to address some of these challenges, for example for wheat (Nature Clim. Change 3, 827-832; 2013). Nevertheless, while there is a growing pool of high-quality research to synthesize (Nature Clim. Change 4, 287-291; 2014), and some general lessons regarding the potential for negative climate change impacts on some crops in some places are beginning to emerge, many questions remain unanswered and/or poorly constrained. One such uncertainty is the role of extreme weather in determining crop yields. The majority of crop impact estimates so far have focused on changes in the average state of the climate, but there is evidence that very hot days can have an important role in determining crop yields (Nature Clim. Change $2,186-189 ; 2012)$ and that changes in the frequency and interaction of adverse weather events is likely to increase the rate of crop failure under climate change (Trnka et al., this issue page 637).

Increased concentrations of atmospheric $\mathrm{CO}_{2}$ can present challenges for crops through influences on the climate system, but the direct effect of more $\mathrm{CO}_{2}$ in the atmosphere is positive for many plants. Photosynthesis in C3-plants - which include important staple crops such as wheat, rice and soy is stimulated by this $\mathrm{CO}_{2}$ fertilization effect. Estimates of the relative magnitude of climate-driven yield loss and fertilizationdriven yield gains indicate that they may roughly cancel each other out (Glob. Environ. Change 14, 53-67; 2004), although whether this will translate into practice remains uncertain. However, a recent meta-analysis (Nature 510, 139-142; 2014) shows that the positive effects on yields are to some extent offset by reduced nutritional value (concentrations of essential minerals and protein) under enhanced $\mathrm{CO}_{2}$ conditions. In a News \& Views (page 540), Müller et al. argue that future analysis needs to move from a quantities-only perspective (focusing on yields and calories) to one that includes nutritional quality. They further argue that this will require consideration of nonstaple crops that may become increasingly important if grains and legumes become increasingly low-quality foods.

People are, of course, not only passive recipients of the environmental fates. Whenever possible we try to anticipate risks and opportunities and alter our plans and actions accordingly. However, quantitative estimates of how much climate impact can be avoided through adaptation of farm systems are difficult. In a Letter (page 610), Moore and Lobell use two different response functions to investigate the spread in climate change impact projections resulting from uncertainty over the rate at which farmers can adopt existing technologies and management solutions. Taking a social perspective on adaptation, Dowd et al. (page 558) show that the combination of strong access to knowledge and weak social ties (social capital) helps to empower individuals to plan and implement novel, rather than incremental, farming strategies.

The extent of climate change impact on crop yield and nutritional value will influence the magnitude of the agricultural challenge, but population growth alone means that yields will need to be increased in the coming decades. This challenge is further exacerbated by growing demand for biofuel crops. There are essentially two ways that such increases in agricultural yield can be achieved globally; by increasing the area under agricultural cultivation (extensification) or by improving the average yield per unit of land already under cultivation (intensification). Both of these choices have implications for the climate. Conversion of natural vegetation, particularly tropical forest, for agriculture leads to loss of carbon that needs to be considered when calculating agricultural contribution to global carbon budgets (Nature Clim. Change 3, 283-287; 2013). Soil carbon can be particularly difficult, and important, to account for (Mello et al., page 605). The loss of natural ecosystems also has clear implications for biodiversity.

Perhaps then intensification is the way to go. Indeed, changes in farm practice may be preferential to farmland expansion for a number of reasons, but intensification too has its impacts on the climate. As Luyssaert et al. show (Nature Clim. Change 4, 389-393; 2014), changes in management can have as great a direct biophysical influence on the climate as changes in land cover. A point reinforced by Jeong et al. (page 615), where a change to double annual cropping practice (wheatmaize) is shown to amplify summertime climate changes over East Asia.

Clearly, managing our agricultural land in such a way as to eliminate hunger, minimize contributions to climate change and environmental degradation while avoiding the worst impacts of unavoidable climate change is a great challenge. A rapidly growing population and increasing demand for biofuels compounds these difficulties. Scientific insights like those presented here will be a vital means of navigating the complexity ahead. 